\title{
CLOSURE OF SINGULAR FOLIATIONS: THE PROOF OF MOLINO'S CONJECTURE
}

\author{
MARCOS M. ALEXANDRINO AND MARCO RADESCHI
}

\begin{abstract}
In this paper we prove the conjecture of Molino that for every singular Riemannian foliation $(M, \mathcal{F})$, the partition $\overline{\mathcal{F}}$ given by the closures of the leaves of $\mathcal{F}$ is again a singular Riemannian foliation.
\end{abstract}

\section{INTRODUCTION}

Given a Riemannian manifold $M$, a singular Riemannian foliation $\mathcal{F}$ on $M$ is, roughly speaking, a partition of $M$ into smooth connected and locally equidistant submanifolds of possibly varying dimension (the leaves of $\mathcal{F}$ ), which is spanned by a family of smooth vector fields. The precise definition, given in Section 2, was suggested by Molino, by combining the concepts of transnormal system of Bolton [5] and of singular foliation by Stefan and Sussmann [13].

A typical example of a singular Riemannian foliation is the decomposition of a Riemannian manifold $M$ into the orbits of an isometric group action $G$ on $M$. Such a foliation is called homogeneous. Another example of foliation is given by the partition of an Euclidean vector bundle $E \rightarrow L$, endowed with a metric connection, into the holonomy tubes around the zero section (cf. Example 2.7). Such a foliation, which we call holonomy foliation, will be a sort-of prototype in the structural results that will appear later on. Holonomy foliations are in general not homogeneous (the zero section $L$ is always a leaf but in general not a homogeneous manifold), however they are locally homogeneous, in the sense that the infinitesimal foliation at every point of $E$ is homogeneous (cf. Sections 2.3 and 2.4). This construction is related to other important types of foliations, like polar foliations [14] or Wilking's dual foliation to the Sharafutdinov projection [15, see Remark 2.8.

In general, the leaves of a singular Riemannian foliation might not be closed, even in the simple cases defined above. In the homogeneous case, consider for example the foliation on the flat torus $T^{2}$ by parallel lines, of irrational slope. These are non closed orbits, of an isometric $\mathbb{R}$-action on $T^{2}$.

Given a (regular) Riemannian foliation $(M, \mathcal{F})$ with non-closed leaves, Molino proved that replacing the leaves of $\mathcal{F}$ with their closure yields a new singular Riemannian foliation $\overline{\mathcal{F}}$. Moreover, he conjectured that the same result should hold true if one starts with a singular Riemannian foliation, and this has become known, in the last decades, as Molino's Conjecture.

Molino proved that the closure $\overline{\mathcal{F}}$ of a singular Riemannian foliation $(M, \mathcal{F})$ is a transnormal system [9], thus leaving to prove that it is a singular foliation as well.

2000 Mathematics Subject Classification. Primary 53C12, Secondary 57R30.

Key words and phrases. Singular Riemannian foliation, linearization, Molino's conjecture.

The first author was partially supported by FAPESP. The second author is part of the DFG project SFB 878: Groups, Geometry \& Actions. 
Moreover, in [10] he suggested a strategy to prove the conjecture for the case of orbit-like foliations, i.e. foliations which, roughly speaking, are locally diffeomorphic to the orbits of some proper isometric group action around each point (cf. Section 2.4). A formal alternative proof in this case can be found in [4]. Molino's conjecture was also proved for polar foliations and then infinitesimally polar foliations in [1] and [3], respectively.

These partial results do not cover every possible foliation. Since the Eighties there are examples of non orbit-like foliations, and in recent years it was shown the existence of a remarkably large class of "infinitesimal" foliations that are neither homogeneous nor polar, the so-called Clifford foliations [12] (these infinitesimal foliations have been shown, however, to have an algebraic nature, cf. [7]). Therefore, it is important to give a complete answer to the conjecture, to fully understand the semi-local dynamic of singular Riemannian foliations.

The goal of this paper is to prove the full Molino's conjecture.

Theorem. (Molino's Conjecture) Let $(M, \mathcal{F})$ be a singular Riemannian foliation on a complete manifold $M$, and let $\overline{\mathcal{F}}=\{\bar{L} \mid L \in \mathcal{F}\}$ be the partition of $M$ into the closures of the leaves of $\mathcal{F}$. Then $(M, \overline{\mathcal{F}})$ is a singular Riemannian foliation.

This result is in fact a direct consequence of the following.

Main Theorem. Let $(M, \mathcal{F})$ be a singular Riemannian foliation, let $L$ be a (possibly not closed) leaf, and let $U$ be an $\epsilon$-neighbourhood around the closure of $L$. Then for $\epsilon$ small enough, there is a metric $\mathrm{g}^{\ell}$ on $U$ and a singular foliation $\widehat{\mathcal{F}}^{\ell}$, such that:

(1) $\left(U, \mathrm{~g}^{\ell}, \widehat{\mathcal{F}}^{\ell}\right)$ is an orbit-like singular Riemannian foliation.

(2) The foliation $\hat{\mathcal{F}}^{\ell}$ coincides with $\mathcal{F}$ on $\bar{L}$.

(3) The closure of $\widehat{\mathcal{F}}^{\ell}$ is contained in the closure of $\mathcal{F}$.

In short, the foliation $\widehat{\mathcal{F}}^{\ell}$ is obtained by first constructing the linearized foliation $\mathcal{F}^{\ell}$ of $\mathcal{F}$ in $U$, which is a subfoliation of $\mathcal{F}$ spanned by the first order approximations, around $L$, of the vector fields tangent to $\mathcal{F}$ (see Section 2.5 for a precise definition). The foliation $\widehat{\mathcal{F}}^{\ell}$ is then obtained from $\mathcal{F}^{\ell}$ by taking the "local closure" of the leaves of $\mathcal{F}^{\ell}$. The foliations $\mathcal{F}, \mathcal{F}^{\ell}, \widehat{\mathcal{F}}^{\ell}$, together with their closures, are then related by the following inclusions:

$$
\begin{aligned}
& \mathcal{F} \supseteq \mathcal{F}^{\ell} \subseteq \widehat{\mathcal{F}}^{\ell} \\
& \text { in in in } \\
& \overline{\mathcal{F}} \supseteq \overline{\mathcal{F}}^{\ell}=\overline{\mathcal{F}}^{\ell}
\end{aligned}
$$

Example 1.1. Consider an Euclidean vector bundle $E$ over a complete Riemannian manifold $L$, with a metric connection $\nabla^{E}$ and a connection metric $g^{E}$ (cf. Example 2.7). Let $H_{p}$ denote the holonomy group of $\left(E, \nabla^{E}\right)$ at $p$, acting by isometries on the Euclidean fiber $E_{p}$, and let $\left(E_{p}, \mathcal{F}_{p}^{0}\right)$ be a singular Riemannian foliation preserved by the $H_{p}$-action. Finally, let $K_{p}$ be the maximal connected group of isometries of $E_{p}$ that fixes each leaf of $\mathcal{F}_{p}^{0}$ as a set.

Letting $\mathcal{F}$ the partition of $E$ into the holonomy translates of the leaves of $\mathcal{F}_{p}^{0}$ (i.e., for every leaf $\mathcal{L} \in \mathcal{F}_{p}^{0}, L_{\mathcal{L}}$ denotes the set of points in $E$ that can be reached via $\nabla^{E}$-parallel translation from a point in $\mathcal{L}$ ), then $\mathcal{F}$ is a singular Riemannian foliation. In this case, the linearized foliation $\mathcal{F}^{\ell}$ is the foliation by the holonomy 
translates of the $K_{p}$-orbits in $E_{p}$, and the local closure of $\widehat{\mathcal{F}}^{\ell}$ is the foliation by the holonomy translates of the $\bar{K}_{p}$-orbits in $E_{p}$, where $\bar{K}_{p}$ denotes the closure of $K_{p}$ in $\mathrm{O}\left(E_{p}\right)$.

This can be restated in the language of groupoids: defining $H$ the holonomy groupoid of the connection $\nabla^{E}$, then $\mathcal{F}=\left\{H(\mathcal{L}) \mid \mathcal{L} \in \mathcal{F}_{p}^{0}\right\}, \mathcal{F}^{\ell}$ is given by the orbits of $H K_{p}$, and its local closure $\hat{\mathcal{F}}^{\ell}$ is given by the orbits of $H \bar{K}_{p}$.

This paper is organized as follows: after a section of preliminaries (Section 2) we show how the Molino's Conjecture follows from the Main Theorem (Section 3). In Section 4 we fix the setup in which we work for the rest of the paper. In Section 5 we define three distributions of the tangent bundle $T U$. We first use these to obtain information on the local structure of $\mathcal{F}^{\ell}$ and define the local closure $\widehat{\mathcal{F}}^{\ell}$ (Section 6) and then to define the metric $\mathrm{g}^{\ell}$ used in the Main Theorem (Section 7). In this final section we also prove the Main Theorem.

\section{ACKNOWLEDGEMENTS}

The authors thank Prof. Lytchak and Prof. Thorbergsson for consistent support.

\section{Preliminaries}

Given a Riemannian manifold $(M, \mathrm{~g})$, a partition $\mathcal{F}$ of $M$ into complete connected submanifolds (the leaves of $\mathcal{F}$ ) is called a transnormal system if geodesics starting perpendicular to a leaf stay perpendicular to all leaves, and a singular foliation if every vector tangent to a leaf can be locally extended to a vector field everywhere tangent to the leaves. A singular Riemannian foliation will be denoted by the triple $(M, \mathrm{~g}, \mathcal{F})$. However, if the Riemannian metric of $M$ is understood, we will drop it and simply write $(M, \mathcal{F})$.

The following notation will be used throughout the rest of the paper. Given a point $p \in M$, the leaf of $\mathcal{F}$ through $p$ will be denoted by $L_{p}$. A small relatively compact open subset $P \subset L$ is called a plaque. The tangent and normal spaces to $L_{p}$ at $p$ are denoted by $T_{p} L_{p}$ and $\nu_{p} L_{p}$, respectively. Given some $\epsilon>0, \nu_{p}^{\epsilon} L_{p}$ denotes the set of vectors $x \in \nu_{p} L_{p}$ with norm $<\epsilon$. If $\epsilon$ is small enough that the normal exponential map exp : $\nu_{p}^{\epsilon} L_{p} \rightarrow M$ is a diffeomorphism onto the image, such image is called a slice of $L_{p}$ at $p$, and it is denoted by $S_{p}$. The slice foliation $\left.\mathcal{F}\right|_{S_{p}}$ denotes the partition of $S_{p}$ into the connected components of the intersections $L \cap S_{p}$, where $L \in \mathcal{F}$.

2.1. Vector fields of a singular Riemannian foliation. We review here the main notations about vector fields of a singular Riemannian foliation.

A vector field $V$ is called vertical if it is tangent to the leaves at each point. The set of smooth vertical vector fields is a Lie algebra, which is denoted by $\mathfrak{X}(M, \mathcal{F})$.

A vector field $X$ is called foliated if its flow takes leaves to leaves or, equivalently, if $[X, V] \in \mathfrak{X}(M, \mathcal{F})$ for every $V \in \mathfrak{X}(M, \mathcal{F})$. Any vertical vector field is foliated, but there are other foliated vector fields. A vector field is called basic if it is both foliated and everywhere normal to the leaves.

2.2. Homothetic Transformation Lemma. One of the most fundamental results in the theory of singular Riemannian foliations is the Homothetic Transformation Lemma. A deeper discussion of this lemma, with proof and applications, can be found in Molino 9, Ch. 6, in particular Lemma 6.1 and Proposition 6.7. 
Let $(M, \mathcal{F})$ be a singular foliation, let $L$ be a leaf of $\mathcal{F}$, and let $P \subset L$ a plaque. Let $\epsilon>0$ be such that the normal exponential map exp : $\nu^{\epsilon} P \rightarrow M$ is a diffeomorphism onto its image $B_{\epsilon}(P)$. For any two radii $r_{1}, r_{2}=\lambda r_{1}$ in $(0, \epsilon)$, it makes sense to define the homothetic transformation

$$
h_{\lambda}: B_{r_{1}}(P) \rightarrow B_{r_{2}}(P), \quad h_{\lambda}(\exp v)=\exp \lambda v .
$$

The leaves of $\mathcal{F}$ intersect $B_{r_{i}}(P), i=1,2$, in plaques that foliate $B_{r_{i}}(P)$. We call $\left.\mathcal{F}\right|_{B_{r_{i}}(P)}$ the foliation of $B_{r_{i}}(P)$ into the path components of such intersections. One has then the following:

Theorem 2.1 (Homothetic Transformation Lemma). The homothetic transformation $h_{\lambda}$ takes the leaves of $\left(B_{r_{1}},\left.\mathcal{F}\right|_{B_{r_{1}}(P)}\right)$ onto the leaves of $\left(B_{r_{2}},\left.\mathcal{F}\right|_{B_{r_{2}}(P)}\right)$.

This result still holds, more generally, if we replace the plaque $P$ by an open subset $B$ of some submanifold $N \subset M$ which is a union of leaves of the same dimension. In this case we consider some $\epsilon>0$ such that $\exp : \nu^{\epsilon} B \rightarrow M$ is a diffeomorphism onto the image $B_{\epsilon}(B)$, and define the homothetic transformation around $W, h_{\lambda}: B_{r}(B) \rightarrow B_{\lambda r}(B)$, as before. In this case, an analogous version of the Homothetic Transformation Lemma applies.

2.3. Infinitesimal foliation. Let $(M, \mathcal{F})$ be a singular Riemannian foliation, $p \in$ $M$ a point, and $S_{p}$ a slice at $p$.

Definition 2.2 (Infinitesimal foliation at $p$ ). The infinitesimal foliation of $\mathcal{F}$ at $p$, denoted by $\left(\nu_{p} L_{p}, \mathcal{F}_{p}\right)$ is defined as the partition of $\nu_{p} L_{p}$ whose leaf at $v \in \nu_{p} L_{p}$ is given by

$$
L_{v}=\left\{w \in \nu_{p} L_{p} \mid \exp _{p} t w \in L_{\left.\exp _{p} t v \quad \forall t>0 \text { small enough }\right\}}\right.
$$

where $L_{\exp _{p} t v}$ denotes the leaf of $\left(S_{p},\left.\mathcal{F}\right|_{S_{p}}\right)$ through $\exp _{p} t v$.

The leaf $L_{v}$ is well defined because, by the Homothetic Transformation Lemma, if $\exp _{p} t_{0} w$ belongs to the same leaf of $\exp _{p} t_{0} v$ for some small $t_{0}$, then $\exp _{p} t w$ belongs to the same leaf of $\exp _{p} t v$ for every $t \in\left(0, t_{0}\right)$. In the following proposition we collect the important facts about infinitesimal foliations that we will need.

Theorem 2.3. Given a singular Riemannian foliation $(M, \mathcal{F})$ and a point $p \in M$ with infinitesimal foliation $\left(\nu_{p} L_{p}, \mathcal{F}_{p}\right)$, then:

(1) The foliation $\left(\nu_{p} L_{p}, \mathcal{F}_{p}\right)$ is a singular Riemannian foliation with respect to the flat metric $\mathrm{g}_{p}$ at $p$.

(2) The normal exponential map $\exp _{p}: \nu_{p}^{\epsilon} L_{p} \rightarrow M$ sends the leaves of $\mathcal{F}_{p}$ to the leaves of $\left(S_{p},\left.\mathcal{F}\right|_{S_{p}}\right)$.

(3) $\left(\nu_{p} L_{p}, \mathcal{F}_{p}\right)$ is invariant under rescalings $r_{\lambda}: \nu_{p} L_{p} \rightarrow \nu_{p} L_{p}, r_{\lambda}(v)=\lambda v$.

Proof. 1) 9], Prop. 6.5.

2) Follows from the definition of infinitesimal foliation, and of slice foliation.

3) Via the exponential map $\exp : \nu^{\epsilon} L_{p} \rightarrow S_{p}$, this corresponds to the Homothetic Transformation Lemma on $S_{p}$.

The following fact will come very useful.

Proposition 2.4. Given singular Riemannian foliations $(M, \mathcal{F}),\left(M^{\prime}, \mathcal{F}^{\prime}\right)$ and a foliated diffeomorphism $\phi: U \rightarrow U^{\prime}$, between open sets $U, U^{\prime}$ of $M, M^{\prime}$ respectively, sending a point $p \in U$ to $p^{\prime} \in U^{\prime}$, the differential of $\phi$ induces a linear, foliated isomorphism $\phi_{*}:\left(\nu_{p} L_{p}, \mathcal{F}_{p}\right) \rightarrow\left(\nu_{p^{\prime}} L_{p^{\prime}}, \mathcal{F}_{p^{\prime}}^{\prime}\right)$. 
Proof. By substituting $\left(M^{\prime}, \mathrm{g}^{\prime}, \mathcal{F}^{\prime}\right)$ with $\left(M, \phi^{*} g^{\prime}, \mathcal{F}\right)$, the problem can be reduced to the case where $M=M^{\prime}, \phi=i d, p=p^{\prime}$, and $\mathcal{F}=\mathcal{F}^{\prime}$ is a singular Riemannian foliation with respect to two metrics, g and $\tilde{g}$. In the following, we will denote with a "tilde" $(\sim)$ every geometric object related to the metric $\tilde{g}$, and without the tilde any geometric object related to g.

Let $S_{p}$ (resp. $\left.\widetilde{S}_{p}\right)$ denote a slice at $p$ with respect to g (resp. $\left.\widetilde{g}\right)$. Consider the set $\left\{X_{1}, \ldots X_{k}\right\} \subset \mathfrak{X}(M, \mathcal{F}), k=\operatorname{dim} L_{p}$, of vector fields such that $\left\{X_{1}(p), \ldots, X_{k}(p)\right\}$ is a basis of $T_{p} L_{p}$. Denote by $\Phi_{i}^{t}$ the flow of $X_{i}$, and define $\Phi_{\left(t_{1}, \ldots t_{k}\right)}=\Phi_{k}^{t_{k}} \circ \ldots \circ \Phi_{1}^{t_{1}}$.

Around $p$, both $S_{p}$ and $\widetilde{S}_{p}$ are transverse to $\operatorname{span}\left(X_{1}, \ldots X_{k}\right)$ and, up to possibly replacing $S_{p}$ and $\widetilde{S}_{p}$ with smaller open subsets, we can assume that for every $q \in S_{p}$ there exists a unique $\tilde{q} \in \widetilde{S}_{p}$ of the form $\tilde{q}=\Phi_{\left(t_{1}, \ldots t_{k}\right)}(q)$. This gives rise to a map $H: S_{p} \rightarrow \widetilde{S}_{p}, H(q)=\tilde{q}$ which is differentiable and, since $q$ and $\tilde{q}$ belong to the same leaf of $\mathcal{F}$, sends the leaves of $\left.\mathcal{F}\right|_{S_{p}}$ to the leaves of $\left.\mathcal{F}\right|_{\widetilde{S}_{p}}$. In other words, there is a foliated diffeomorphism $H:\left(S_{p},\left.\mathcal{F}\right|_{S_{p}}\right) \rightarrow\left(\widetilde{S}_{p},\left.\mathcal{F}\right|_{\widetilde{S}_{p}}\right)$.

Consider the composition $\psi$ of foliated diffeomorphisms

$$
\left(\nu_{p}^{\epsilon} L_{p}, \mathcal{F}_{p}\right) \stackrel{\exp _{p}}{\longrightarrow}\left(S_{p},\left.\mathcal{F}\right|_{S_{p}}\right) \stackrel{H}{\longrightarrow}\left(\widetilde{S}_{p},\left.\mathcal{F}\right|_{\widetilde{S}_{p}}\right) \stackrel{\text { exp }}{\longrightarrow}\left(\tilde{\nu}_{p}^{\epsilon} L_{p}, \widetilde{\mathcal{F}}_{p}\right) .
$$

For any $\lambda \in(0,1)$, one can define a new foliated diffeomorphism

$$
\psi_{\lambda}:\left(\nu_{p}^{\epsilon / \lambda} L_{p}, \mathcal{F}_{p}\right) \rightarrow\left(\tilde{\nu}_{p}^{\epsilon / \lambda} L_{p}, \widetilde{\mathcal{F}}_{p}\right), \quad \psi_{\lambda}(v)=\frac{1}{\lambda} \psi(\lambda v) .
$$

As $\lambda \rightarrow 0$, the maps $\psi_{\lambda}$ converge to the differential $d_{0} \psi$ of $\psi$ at 0 . This is an invertible linear map (in particular a diffeomorphism) and, as a limit of foliated maps, it is itself foliated. Therefore, the map

$$
\phi_{*}:=d_{0} \psi:\left(\nu_{p} L, \mathcal{F}_{p}\right) \longrightarrow\left(\tilde{\nu}_{p} L, \widetilde{\mathcal{F}}_{p}\right)
$$

satisfies the statement of the proposition.

Remark 2.5. Given a singular Riemannian foliation $(M, \mathcal{F})$ and a submanifold $N \subset$ $M$ which is a union of leaves of the same dimension, the infinitesimal foliation at a point $p \in M$ splits as a product $\left(\nu_{p}\left(L_{p}, N\right) \times \nu_{p} N,\{\right.$ pts. $\left.\} \times\left.\mathcal{F}_{p}\right|_{\nu_{p} N}\right)$, where $\nu_{p}\left(L_{p}, N\right)=\nu_{p} L_{p} \cap T_{p} N$. In this case, the foliation $\left(\nu_{p} N,\left.\mathcal{F}_{p}\right|_{\nu_{p} N}\right)$ is the "essential part" of the infinitesimal foliation $\left(\nu_{p} L_{p}, \mathcal{F}_{p}\right)$. By abuse of notation, we will call the foliation $\left(\nu_{p} N,\left.\mathcal{F}_{p}\right|_{\nu_{p} N}\right)$ infinitesimal foliation at $p$ as well, and denote it by $\mathcal{F}_{p}$.

Given a singular Riemannian foliation $(M, \mathcal{F})$ and a point $p \in M$, the infinitesimal foliation $\left(\nu_{p} L_{p}, \mathcal{F}_{p}\right)$ at $p$ contains the origin as a leaf of $\mathcal{F}_{p}$. Based on this fact, we make the following definition.

Definition 2.6 (Infinitesimal foliation). An infinitesimal foliation is a singular Riemannian foliation $(V, \mathcal{F})$ on an Euclidean vector space, with the origin $\{0\}$ being a 0 -dimensional leaf.

2.4. Homogeneous and orbit like foliations. A singular Riemannian foliation $(M, \mathcal{F})$ is called homogeneous (sometimes Riemannian homogeneous) if there exists a connected Lie group $G$ acting by isometries on $M$, whose orbits are precisely the leaves of $\mathcal{F}$. Furthermore, a singular Riemannian foliation $(M, \mathcal{F})$ is called orbitlike if at every point $p \in M$, the infinitesimal foliation $\left(\nu_{p} L_{p}, \mathcal{F}_{p}\right)$ is closed and homogeneous. 
Example 2.7 (Holonomy foliations). An example of orbit like foliation, which will be useful to keep in mind later on, can be constructed as follows. Consider a Riemannian manifold $L$, and an Euclidean vector bundle $E$ over $L$, that is, a vector bundle over $L$ with an inner product $\langle,\rangle_{p}$ on each fiber $E_{p}, p \in L$. Let $\nabla^{E}$ be a metric connection on $E$, i.e. a connection on $E$ such that, for every vector field $X$ on $L$ and sections $\xi, \eta$ of $E$, one has

$$
X\langle\xi, \eta\rangle=\left\langle\nabla_{X}^{E} \xi, \eta\right\rangle+\left\langle\xi, \nabla_{X}^{E} \eta\right\rangle .
$$

Given $\left(E, \nabla^{E}\right)$, there is an induced Riemannian metric $\mathrm{g}^{E}$ on $E$, called connection metric. Moreover, $\nabla^{E}$ induces a parallel transport on $E$ : given $\xi \in E_{p}$ and a curve $\gamma:[0,1] \rightarrow L$ with $\gamma(0)=p$, there exists a unique lift $\xi(t), t \in[0,1]$ with $\xi(0)=\xi$ such that $\nabla_{\gamma^{\prime}(t)}^{E} \xi(t)=0$ for every $t \in[0,1]$. On $E$ one can now define a foliation $\mathcal{F}^{E}$, by declaring two vectors $\xi, \eta \in E$ in the same leaf if they can be connected to one another via a composition of parallel transports. The leaves of $\mathcal{F}^{E}$ are usually referred to as the holonomy tubes around the zero section $L \subset E$, and they define a singular Riemannian foliation on $\left(E, \mathrm{~g}^{E}\right)$. Moreover, the infinitesimal foliation at any point of $E$ is homogeneous: in fact, for any point $p$ along the zero section $L$, one can first construct the holonomy group $H_{p}$ of the connection $\nabla^{E}$, which acts by isometries on the fiber $E_{p}$ and whose orbits are precisely the leaves of the infinitesimal foliation of $\mathcal{F}^{E}$ at $p$. Similarly, the infinitesimal foliation at a point $\xi \in E_{p}$ is given by the orbits in $\nu_{\xi} L_{\xi}$ of the stabilizer $H_{\xi} \subset H_{p}$ of $\xi$. The foliation $\mathcal{F}^{E}$ coincides with its own linearization with respect to the zero section (see definition in Section 2.5). Moreover, if the leaves of $\mathcal{F}^{E}$ are closed then $\left(E, \mathrm{~g}^{E}, \mathcal{F}^{E}\right)$ is an orbit-like foliation.

Remark 2.8. When $L \subset M$ is a submanifold of somewhat special geometry, the holonomy foliation on the normal bundle $E$ of $L$, endowed with the Levi-Civita connection, induces via the normal exponential map a foliation on the whole of $M$. For example, if $L$ has parallel focal structure, then the induced foliation on $M$ is a polar foliation [14. If $M$ is a complete, non-compact manifold with sectional curvature $\geq 0$ and $L$ is a soul of $M[6]$, then the induced foliation on $M$ is Wilking's dual foliation to the Sharafutdinov projection [15].

Although in principle the property of being orbit-like might depend on the metric, the following proposition shows in fact that being orbit like is invariant under foliated diffeomorphisms.

Proposition 2.9. The following hold:

(1) Given a foliated linear isomorphism $\varphi:(V, \mathcal{F}) \rightarrow\left(V^{\prime}, \mathcal{F}^{\prime}\right)$ between infinitesimal foliations, $(V, \mathcal{F})$ is homogeneous if and only if $\left(V^{\prime}, \mathcal{F}^{\prime}\right)$ is homogeneous.

(2) Given a foliated diffeomorphism $\phi:(M, \mathcal{F}) \rightarrow\left(M^{\prime}, \mathcal{F}^{\prime}\right)$ between singular Riemannian foliations, $(M, \mathcal{F})$ is orbit-like if and only if $\left(M^{\prime}, \mathcal{F}^{\prime}\right)$ is orbitlike.

Proof. 1) By the symmetric roles of $V$ and $V^{\prime}$, it is enough to show that if $(V, \mathcal{F})$ is homogeneous, so is $\left(V^{\prime}, \mathcal{F}^{\prime}\right)$. Suppose that $(V, \mathcal{F})$ is homogeneous, and therefore the foliation $\mathcal{F}$ is spanned by Killing fields. Recall that a vector field $X$ on an Euclidean space $(V, \mathrm{~g})$ is Killing if and only if is of the form $X(v)=A v$, where $A$ is a skew symmetric endomorphism of $V$, in the sense that $\mathrm{g}(A v, v)=0$ for every $v \in V$. Letting $\left\{X_{1}, \ldots X_{k}\right\}$ denote a set of Killing fields on $V$ spanning the foliation $\mathcal{F}$, 
the set $\left\{Y_{1}, \ldots Y_{k}\right\}$ with $Y_{i}\left(v^{\prime}\right)=\varphi_{*}\left(X_{i}\left(\varphi^{-1}\left(v^{\prime}\right)\right)\right)$ spans the foliation $\mathcal{F}^{\prime}$ as well. Since $\varphi$ is a linear map and the vector fields $X_{i}$ are linear, it follows that $Y_{i}$ can be written as $Y_{i}\left(v^{\prime}\right)=B_{i} v^{\prime}$ for some endomorphism $B_{i}: V^{\prime} \rightarrow V^{\prime}, i=1 \ldots k$. Since $\left(V^{\prime}, \mathrm{g}^{\prime}, \mathcal{F}^{\prime}\right)$ is a singular Riemannian foliation, the leaf $L_{v^{\prime}}$ through $v^{\prime}$ lies in a distance sphere from the origin, and in particular $\mathrm{g}^{\prime}\left(T_{v^{\prime}} L_{v^{\prime}}, v^{\prime}\right)=0$. Since $Y_{i}\left(v^{\prime}\right)$ is tangent to $L_{v^{\prime}}$, it follows that

$$
0=\mathrm{g}^{\prime}\left(Y_{i}\left(v^{\prime}\right), v^{\prime}\right)=\mathrm{g}\left(B_{i} v^{\prime}, v^{\prime}\right)
$$

In other words, $B_{i}$ is skew-symmetric and thus $Y_{i}$ is a Killing field as well. Therefore the foliation $\left(V^{\prime}, \mathcal{F}^{\prime}\right)$ is spanned by Killing vector fields, hence it is homogeneous as well.

2) Up to exchanging the roles of $M$ and $M^{\prime}$, it is enough to show that if $(M, \mathcal{F})$ is orbit-like, so is $\left(M^{\prime}, \mathcal{F}^{\prime}\right)$. Fixing a point $p \in M$, Proposition 2.4 states that the foliated diffeomorphism $\phi$ induces a foliated linear isomorphism $\phi_{*}:\left(\nu_{p} L_{p}, \mathcal{F}_{p}\right) \longrightarrow$ $\left(\nu_{p^{\prime}} L_{p^{\prime}}, \mathcal{F}_{p^{\prime}}\right)$, where $p^{\prime}=\phi(p)$. Since $(M, \mathcal{F})$ is orbit-like, it follows that $\left(\nu_{p} L_{p}, \mathcal{F}_{p}\right)$ is closed and homogeneous. From the first point above it follows that $\left(\nu_{p^{\prime}} L_{p^{\prime}}, \mathcal{F}_{p^{\prime}}\right)$ is homogeneous as well, and by the continuity of $\phi_{*}$ one has that $\left(\nu_{p^{\prime}} L_{p^{\prime}}, \mathcal{F}_{p^{\prime}}\right)$ is closed. Since $p^{\prime}$ was chosen arbitrarily, it follows that $\left(M^{\prime}, \mathcal{F}^{\prime}\right)$ is orbit-like.

2.5. Linearization, and linearized foliation. Let $(M, \mathcal{F})$ be a singular Riemannian foliation, $B \subset M$ a submanifold saturated by leaves, and $U \in M$ an $\epsilon$-tubular neighbourhood of $B$ with metric projection $\mathrm{p}: U \rightarrow B$. Given a vector field $V$ in $U$ tangent to the leaves of $\mathcal{F}$, it is possible to produce a new vector field $V^{\ell}$, called the linearization of $V$ with respect to $B$, as follows:

$$
V^{\ell}=\lim _{\lambda \rightarrow 0}\left(h_{\lambda}^{-1}\right)_{*}\left(\left.V\right|_{h_{\lambda}(U)}\right)
$$

where $h_{\lambda}: U \rightarrow U$ denotes the homothetic transformation around $B$. From [8, Prop. 5, the linearization $V^{\ell}$ is a smooth vector field invariant under the homothetic transformation $h_{\lambda}$, and it coincides with $V$ along $B$.

On $U$, consider the module $\mathfrak{X}(U, \mathcal{F})^{\ell}$ given by the linearization, with respect to $B$, of the vector fields in $\mathfrak{X}(U, \mathcal{F})$ :

$$
\mathfrak{X}(U, \mathcal{F})^{\ell}=\left\{V^{\ell} \mid V \in \mathfrak{X}(U, \mathcal{F})\right\} .
$$

Let $\mathrm{D}$ the pseudogroup of local diffeomorphisms of $U$, generated by the flows of linearized vector fields, and let $\left(U, \mathcal{F}^{\ell}\right)$ the partition of $U$ into the orbits of diffeomorphisms in D. By Sussmann [13, Thm. 4.1], such orbits are (possibly noncomplete) smooth submanifolds of $M$. Moreover, as noted By Molino 9, Lem. 6.3], this foliation is spanned, at each point, by the vector fields in $\mathfrak{X}^{\ell}(U, \mathcal{F})$.

We call $\left(U, \mathcal{F}^{\ell}\right)$ the linearized foliation of $\mathcal{F}$ with respect to $B$. We will show, later, that the leaves of the linearized foliation are actually complete, and have a particularly nice local structure (cf. Section 6).

Given a point $p \in B$, define $U_{p}=\mathrm{p}^{-1}(p) \subset U$ and let $\mathcal{F}_{p}\left(\right.$ resp. $\left.\left(\mathcal{F}^{\ell}\right)_{p}\right)$ denote the partition of $U_{p}$ into the connected components of $L \cap U_{p}$, as $L$ ranges through the leaves of $\mathcal{F}$ (resp. $\mathcal{F}^{\ell}$ ). If $U_{p}$ is given the flat metric $\mathrm{g}_{p}$ of $\nu_{p} B$ via the exponential map $\exp _{p}: \nu_{p}^{\epsilon} B \rightarrow U_{p}$, then $\mathcal{F}_{p}$ corresponds to the infinitesimal foliation at $p$ (cf. Remark 2.5) which justifies the notation of $\mathcal{F}_{p}$ for this foliation. Furthermore, as noted in [9, Sec. 6.4], $\left(\mathcal{F}^{\ell}\right)_{p}$ is given by the linearization of $\left(U_{p}, \mathrm{~g}_{p}, \mathcal{F}_{p}\right)$ with respect to the origin. In other words, $\left(\mathcal{F}^{\ell}\right)_{p}=\left(\mathcal{F}_{p}\right)^{\ell}$ and it makes sense to denote this 
foliation simply by $\mathcal{F}_{p}^{\ell}$. Moreover, letting $\mathrm{O}\left(\mathcal{F}_{p}\right)$ denote the Lie group of (linear) isometries of $\left(U_{p}, \mathrm{~g}_{p}\right)$ sending every leaf to itself, one has:

Proposition 2.10. The foliation $\left(U_{p}, \mathcal{F}_{p}^{\ell}\right)$ is homogeneous, given by the orbits of the identity component $H_{p}$ of $\mathrm{O}\left(\mathcal{F}_{p}\right)$.

Proof. We identify here $U_{p}$ with a neighbourhood of the origin in $\nu_{p} B$ via the exponential map, and we think of $\mathcal{F}_{p}^{\ell}$ as the linearization of $\mathcal{F}_{p}$.

Given a vector field $V \in \mathfrak{X}\left(U_{p}, \mathcal{F}_{p}\right)$, its linearization $V^{\ell}$ is linear, in the sense that $V_{p}^{\ell}=A \cdot p$ for some $A \in \operatorname{End}\left(U_{p}\right)$. Since $\mathcal{F}_{p}$ is a singular Riemannian foliation, the leaves are tangent to the distance spheres around the origin and therefore perpendicular to the radial directions from the origin: $\left\langle V_{p}^{\ell}, p\right\rangle=0$. In other words, $V_{p}^{\ell}=A \cdot p$ with $A$ skew symmetric, which implies that the flow of $V^{\ell}$ is an isometry of $U_{p}$. Moreover, since $V^{\ell}$ is everywhere tangent to the leaves of $\mathcal{F}_{p}^{\ell}$, the flow of $V^{\ell}$ is a 1-parameter group in $H_{p}$, moving every leaf of $\left(\mathcal{F}_{p}\right)^{\ell}$ to itself. In particular, the orbits of $H_{p}$ are contained in the leaves of $\left(\mathcal{F}_{p}\right)^{\ell}$.

However, by definition of $H_{p}$, the tangent space of a $H_{p}$-orbit through a point $q \in U_{p}$ is given by

$$
T_{q}\left(H_{p} \cdot q\right)=\left\{W_{q} \mid W \text { Killing vector field tangent to the leaves of } \mathcal{F}_{p}\right\}
$$

and such vector fields coincide precisely with the vector fields in $\mathfrak{X}\left(U_{p}, \mathcal{F}_{p}\right)^{\ell}$. Therefore, $H_{p} \cdot q$ is the integral manifold of $\mathfrak{X}\left(U_{p}, \mathcal{F}_{p}\right)^{\ell}$ through $q$.

\section{Molino's conjecture, assuming the Main Theorem}

Before proving the Main Theorem, we show how Molino's Conjecture follows from it as a corollary.

Proof of Molino's Conjecture. Let $(M, \mathcal{F})$ be a singular Riemannian foliation, and let $\overline{\mathcal{F}}$ denote the closure of $\mathcal{F}$. Molino himself proved that $\overline{\mathcal{F}}$ is a partition into complete smooth closed submanifolds, and that $\overline{\mathcal{F}}$ is a transnormal system. Therefore, in order to prove the conjecture, it is enough to show that for any leaf $L \in \mathcal{F}$ with closure $\bar{L}$ and any vector $v \in \nu(L, \bar{L}):=\nu L \cap T \bar{L}$, there exists a smooth extension of $v$ to a vector field $V$ everywhere tangent to the leaves of $\overline{\mathcal{F}}$.

Let $U$ be a tubular neighbourhood of $\bar{L}$, and let $\left(U, \widehat{\mathcal{F}}^{\ell}\right)$ be the foliation satisfying the Main Theorem. Since $\widehat{\mathcal{F}}^{\ell}$ coincides with $\mathcal{F}$ along $\bar{L}$, it follows that $L$ is a leaf of $\widehat{\mathcal{F}}^{\ell}$ as well. Since $\widehat{\mathcal{F}}^{\ell}$ is an orbit-like foliation, by Theorem 1.6 of [4, given $v \in \nu(L, \bar{L})$ there is a vector field $V$ extending $v$ which is tangent to the closure of $\widehat{\mathcal{F}}^{\ell}$. Since this closure is contained in $\overline{\mathcal{F}}$, it follows that $V$ is also tangent to $\overline{\mathcal{F}}$ and this ends the proof of the conjecture.

\section{THE SETUP}

Fix a leaf $L$, and distance tube $U=B_{\epsilon}(L)$ around $\bar{L}$. Using the normal exponential map exp : $\nu \bar{L} \rightarrow M, U$ can be identified with the $\epsilon$-tube $\nu^{\epsilon} \bar{L}$ around the zero section. By the Homothetic Transformation Lemma, the pull-back foliation $\exp ^{-1} \mathcal{F}$ on $\nu^{\epsilon} \bar{L}$ is invariant under the rescalings $r_{\lambda}: \nu^{\epsilon} \bar{L} \rightarrow \nu^{\epsilon} \bar{L}, r_{\lambda}(p, v)=(p, \lambda v)$ for any $\lambda \in(0,1)$.

For this reason, in the following sections we will be considering the (slightly more general) setup: 
- $U$ is the $\epsilon$-tube around the zero section of some Euclidean vector bundle $E \rightarrow B$ (in our case $B=\bar{L}$ ), with projection $\mathrm{p}: U \rightarrow B$.

- $\mathrm{g}$ is a Riemannian metric on $U$ with the same radial function as the Euclidean metric on each fiber of $E$.

- $(U, \mathrm{~g}, \mathcal{F})$ is a singular Riemannian foliation on $U$, invariant under rescalings $r_{\lambda}$. In particular, the zero-section $B$ is saturated by leaves and the projection $\mathrm{p}$ sends leaves onto leaves.

- The restriction $\mathcal{F}_{B}=\left.\mathcal{F}\right|_{B}$ is a regular Riemannian foliation.

- For every leaf $L \subseteq B$ and any point $p \in L$, the normal exponential map $\nu_{p}^{\epsilon} L \rightarrow U$ is an embedding.

\section{Three Distributions}

Let $(U, \mathrm{~g}, \mathcal{F}), \mathrm{p}: U \rightarrow B$ be as in Section 4 . In order to prove the Main Theorem, it is first needed to produce a nicer metric on $U$, and for this we first need to split the tangent space of $U$ into three components. The first, $\mathcal{K}=\operatorname{ker} \mathrm{p}_{*}$, is the distribution tangent to the fibers of $\mathrm{p}$. For the remaining two notice that, since the foliation $\left(B, \mathcal{F}_{B}\right)$ is regular, the tangent bundle $T B$ splits into a tangent and a normal part to the foliation: $T B=\left.\left.T \mathcal{F}\right|_{B} \oplus \nu \mathcal{F}\right|_{B}$. The last two distributions will be constructed as (appropriately chosen) extensions $\mathcal{T}$ and $\mathcal{N}$ of $\left.T \mathcal{F}\right|_{B}$ and $\left.\nu \mathcal{F}\right|_{B}$ respectively, to the whole of $U$.

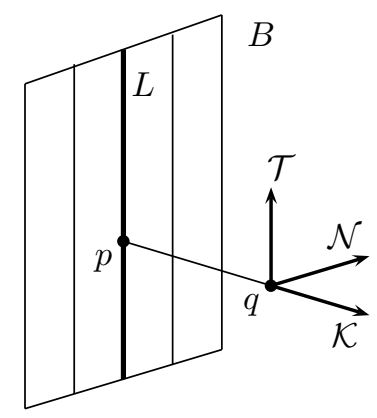

Figure 1. The distributions at $q$.

5.1. The distribution $\mathcal{T}$. From [2] there exists a distribution $\widehat{\mathcal{T}}$ of $\left.\operatorname{rank} \operatorname{dim} \mathcal{F}\right|_{B}$, which extends $\left.T \mathcal{F}\right|_{B}$ and is everywhere tangent to the leaves of $\mathcal{F}$.

The distribution $\mathcal{T}$ is simply defined as the linearization of $\widehat{\mathcal{T}}$ with respect to $B$, as follows: consider a family of vector fields $\left\{V_{\alpha}\right\}_{\alpha}$ spanning $\widehat{\mathcal{T}}$. Since $\left.\widehat{\mathcal{T}}\right|_{B}$ is tangent to $B$, the vector fields $V_{\alpha}$ lie tangent to $B$ as well and therefore it makes sense to consider their linearization $V_{\alpha}^{\ell}$ with respect to $B$. By the properties of the linearization, these linearized vector fields still span a smooth distribution of the same rank as $\widehat{\mathcal{T}}$, which we call $\mathcal{T}$. 
5.2. The distribution $\mathcal{N}$. At each point $q \in U$ with $\mathrm{p}(q)=p$, the slice $S_{p}=$ $\exp _{p}\left(\nu_{p}^{\epsilon} L_{p}\right)$ contains $q$ as well as the whole p-fiber $U_{p}$ through $p$. In particular, $\mathcal{K}_{q}$ lies tangent to $S_{p}$. Moreover, $S_{p}$ comes equipped with a flat metric $\mathrm{g}_{p}$, inherited from the metric on $\nu_{p} L$ via the diffeomorphism $\exp _{p}: \nu^{\epsilon} L_{p} \rightarrow S_{p}$.

Define $\widehat{\mathcal{N}}_{q}$ as the subspace of $T_{q} S_{p}$ which is $g_{p}$-orthogonal to $\mathcal{K}_{q}$. Finally, define $\mathcal{N}$ as the linearization of $\widehat{\mathcal{N}}$, as defined in the previous section.

The distributions $\widehat{\mathcal{N}}$ and $\mathcal{N}$ satisfy the following property:

Proposition 5.1. For every smooth $\mathcal{F}_{B}$-basic vector field $X_{0}$ along a plaque $P$ in $B$ there exists a smooth extension $X$ to an open set of $U$ such that

(1) $X$ is foliated and tangent to $\widehat{\mathcal{N}}$.

(2) The linearization $X^{\ell}$ of $X$ with respect to $B$ is tangent to $\mathcal{N}$, and it is foliated with respect to both $\mathcal{F}$ and $\mathcal{F}^{\ell}$.

Proof. 1) Fix a leaf $L$ in $B$, a plaque $P \subset L$ and a parametrization

$$
\varphi:(-1,1)^{k} \rightarrow P \subset L,
$$

where $k=\operatorname{dim} \mathcal{F}_{B}$. We first show that there exists a small neighbourhood of $P$ in $U$, on which any $\mathcal{F}_{B}$-basic vector field $X_{0}$ along $P$ can be extended to a foliated vector field $X^{\prime}$, whose restriction to $\mathrm{p}^{-1}(P)$ is tangent to $\widehat{\mathcal{N}}$.

Let $\partial_{y_{1}}, \ldots, \partial_{y_{k}}$ be coordinate vector fields on $P$, and let $Y_{1}, \ldots Y_{k}$ denote vector fields, linearized with respect to $L$, that extend $\partial_{y_{1}}, \ldots \partial_{y_{k}}$ to a neighbourhood of $P$ in $U$. There is a foliated diffeomorphism

$$
\begin{aligned}
F:\left(P \times S_{p}, P \times \mathcal{F}_{S_{p}}\right) & \longrightarrow(U, \mathcal{F}) \\
\left(\varphi\left(y_{1}, \ldots y_{k}\right), q\right) & \longmapsto \Phi_{k}^{y_{k}} \circ \ldots \circ \Phi_{1}^{y_{1}}(q)
\end{aligned}
$$

where $\Phi_{i}^{y_{i}}$ is the flow of $Y_{i}$, after time $y_{i}$.

Furthermore, the foliation $\left(P \times S_{p}, P \times \mathcal{F}_{S_{p}}\right)$ locally splits as

$$
\left(P \times \nu_{p}(L, B) \times \nu_{p} B, P \times\{\text { pts. }\} \times\left.\mathcal{F}\right|_{\nu_{p} B}\right)
$$

where $\nu(L, B)=\nu L \cap T B$. Moreover, if $S_{p}$ is endowed with the Euclidean metric $\mathrm{g}_{p}$ on $\nu_{p} L$, the splitting $S_{p}=\nu_{p}(L, B) \times \nu_{p} B$ is in fact Riemannian.

The map $F$ satisfies the following:

- The set $P \times\{0\} \times \nu_{p}^{\epsilon} B$ is sent to $\mathrm{p}^{-1}(P)=\left.\nu^{\epsilon} B\right|_{P}$.

- The set $P \times \nu_{p}^{\epsilon}(L, B) \times\{0\}$ is sent to a neighbourhood of $P$ in $B$.

- Since $F$ is defined via linearized vector fields, each fiber $\left\{p^{\prime}\right\} \times S_{p} \subset P \times S_{p}$ is sent, via $F$, to the slice $S_{p^{\prime}}$, isometrically with respect to the flat metrics on $S_{p}$ and $S_{p^{\prime}}$ (cf. [8]).

From the last point, it follows that the distribution of $P \times \nu_{p}(L, B) \times \nu_{p} B$ tangent to the second factor is sent, along $\left.\nu^{\epsilon} B\right|_{P}$, precisely to the distribution $\widehat{\mathcal{N}}$.

Any $\mathcal{F}_{B}$-basic vector field $X_{0}$ along $P$ corresponds, via $F$, to a vector field along $P \times\{0\} \times\{0\}$ of the form $\left(0, x_{0}, 0\right)$ where $x_{0} \in \nu_{p}(L, B)$ is a fixed vector. One can clearly extend such a vector field to the foliated vector field $X^{\prime}=F_{*}\left(0, x_{0}, 0\right)$. Since $F$ is a foliated map, the vector field $X^{\prime}$ is a foliated vector field, whose restriction to $B$ is tangent to $B$ by the second point above. Moreover, by the discussion above the restriction of $X^{\prime}$ to $\mathrm{p}^{-1}(P)$ is tangent to $\widehat{\mathcal{N}}$.

This proves the first claim, made at the beginning of the proof. In particular, since the plaque $P$ was chosen arbitrarily, this shows that the distribution $\widehat{\mathcal{N}}$ is foliated: that is, given a vector $y$ tangent to $\widehat{\mathcal{N}}$ at a point $q$, there exists a foliated 
extension $Y$ along a plaque containing $q$ which is everywhere tangent to $\widehat{\mathcal{N}}$. It is easy to see that $\mathcal{K}$ and $\mathcal{T}$ are foliated as well. In particular, given the foliated vector field $X^{\prime}$, the (unique) decomposition

$$
X^{\prime}=X_{\mathcal{K}}^{\prime}+X_{\mathcal{T}}^{\prime}+X_{\widehat{\mathcal{N}}}^{\prime}, \quad X_{\mathcal{K}}^{\prime} \in \mathcal{K}, \quad X_{\mathcal{T}}^{\prime} \in \mathcal{T}, \quad X_{\widehat{\mathcal{N}}}^{\prime} \in \widehat{\mathcal{N}}
$$

produces three vector fields $X_{\mathcal{K}}^{\prime}, X_{\mathcal{T}}^{\prime}, X_{\widehat{\mathcal{N}}}^{\prime}$ which are foliated. In particular, the vector field $X=\left(X^{\prime}\right)_{\widehat{\mathcal{N}}}$ is foliated, everywhere tangent to $\widehat{\mathcal{N}}$, and it extends $X_{0}=$ $\left(X_{0}\right)_{\widehat{\mathcal{N}}}$ to an open set of $U$, as we needed to show.

2) Since $X$ is tangent to $\widehat{\mathcal{N}}$, its linearization $X^{\ell}$ is tangent to the linearization of $\widehat{\mathcal{N}}$, which is $\mathcal{N}$. Moreover, since $X$ is foliated and $r_{\lambda}: U \rightarrow U$ is a foliated map, $X^{\ell}=\lim _{\lambda \rightarrow 0}\left(r_{\lambda}^{-1}\right)_{*} X \circ r_{\lambda}$ is foliated as well. Finally, since $X$ is foliated, for every vector field $V$ tangent to $\mathcal{F}$ one has that $[X, V]$ is also tangent to $\mathcal{F}$. Since $r_{\lambda}$ is a diffeomorphism, one computes

$$
\begin{aligned}
{\left[X^{\ell}, V^{\ell}\right] } & =\lim _{\lambda \rightarrow 0}\left[\left(r_{\lambda}^{-1}\right)_{*} X \circ r_{\lambda},\left(r_{\lambda}^{-1}\right)_{*} V \circ r_{\lambda}\right] \\
& =\lim _{\lambda \rightarrow 0}\left(r_{\lambda}^{-1}\right)_{*}[X, V] \circ r_{\lambda} \\
& =[X, V]^{\ell} .
\end{aligned}
$$

Since the linearization $V^{\ell}$ are precisely the vector fields generating $\mathcal{F}^{\ell}$, it follows from the equation above that $\left[X^{\ell}, V^{\ell}\right]$ is tangent to $\mathcal{F}^{\ell}$ whenever $V^{\ell}$ is, and therefore $X^{\ell}$ is foliated with respect to $\mathcal{F}^{\ell}$.

\section{Structure of $\mathcal{F}^{\ell}$, ANd the local Closure $\widehat{\mathcal{F}}^{\ell}$}

Using the extensions $X^{\ell}$ defined in Proposition 5.1, one can prove the following:

Proposition 6.1. Around any point $p \in B$ there is a neighbourhood $W$ of $p$ in $B$ such that $\left(\mathrm{p}^{-1}(W),\left.\mathcal{F}^{\ell}\right|_{\mathrm{p}^{-1}(W)}\right)$ is foliated diffeomorphic to a product

$$
\left(\mathbb{D}^{k} \times \mathbb{D}^{m-k} \times U_{p}, \mathbb{D}^{k} \times\{p t s .\} \times \mathcal{F}_{p}^{\ell}\right)
$$

where $k=\left.\operatorname{dim} \mathcal{F}\right|_{B}$ and $m=\operatorname{dim} B$.

Proof. Let $W$ be a coordinate neighbourhood of $B$ around $p$, with a foliated diffeomorphism $\varphi:\left(W,\left.\mathcal{F}\right|_{W}\right) \rightarrow\left(\mathbb{D}^{k} \times \mathbb{D}^{m-k}, \mathbb{D}^{k} \times\{p t s\}.\right)$. Let $\frac{\partial}{\partial y_{1}}, \ldots \frac{\partial}{\partial y_{k}}$ denote a basis of vector fields in $W$ tangent to the leaves of $\left.\mathcal{F}\right|_{W}$, and let $V_{1}, \ldots V_{k}$ denote vector fields on $\mathrm{p}^{-1}(W)$, linearized with respect to $B$, extending $\frac{\partial}{\partial y_{i}}, i=1, \ldots k$ and spanning the foliation $\mathcal{T}$. Similarly, let $\frac{\partial}{\partial x_{1}}, \ldots \frac{\partial}{\partial x_{m-k}}$ denote a basis of basic vector fields in $W$ normal to the leaves, and let $X_{1}^{\ell}, \ldots X_{m-k}^{\ell}$ denote linearized vector fields in $\pi^{-1}(W)$ defined as in Proposition 5.1 extending the vectors $\frac{\partial}{\partial x_{i}}$, $i=1, \ldots m-k$. Finally, define $\Phi_{i}^{t}$ and $\Psi_{i}^{t}$ the flows of $V_{i}$ and $X_{i}^{\ell}$ respectively, after time $t$, and let

$$
\begin{aligned}
G: \mathbb{D}^{k} \times \mathbb{D}^{m-k} \times U_{p} & \longrightarrow \mathrm{p}^{-1}(W) \\
\left(\left(t_{1}, \ldots, t_{k}\right),\left(s_{1}, \ldots, s_{m-k}\right), q\right) & \longmapsto \Phi_{k}^{t_{k}} \circ \ldots \circ \Phi_{1}^{t_{1}} \circ \Psi_{m-k}^{s_{m-k}} \circ \ldots \circ \Psi_{1}^{s_{1}}(q) .
\end{aligned}
$$

Since the $V_{i}$ and $X_{i}^{\ell}$ are linearized, they take fibers of $\mathbb{D}^{k} \times \mathbb{D}^{m-k} \times U_{p} \rightarrow \mathbb{D}^{k} \times \mathbb{D}^{m-k}$ to fibers of $\mathrm{p}: \mathrm{p}^{-1}(W) \rightarrow W$. Since the flows $\Psi_{i}$ send the leaves of $\mathcal{F}^{\ell}$ to leaves, and the flows $\Phi_{i}$ take leaves of $\mathcal{F}^{\ell}$ to themselves, the leaves of $\mathbb{D}^{k} \times\left(\mathbb{D}^{m-k},\{\right.$ pts. $\left.\}\right) \times$ $\left(U_{p}, \mathcal{F}_{p}^{\ell}\right)$ are sent into the leaves of $\mathcal{F}^{\ell}$. Since the differential $d G$ is invertible at 
$(0,0, p) \in \mathbb{D}^{k} \times \mathbb{D}^{m-k} \times U_{p}$, it is a diffeomorphism around $G(0,0, p)=p$ and, by dimensional reasons, the leaves of $\left(\mathbb{D}^{k} \times \mathbb{D}^{m-k} \times U_{p}, \mathbb{D}^{k} \times\{\right.$ pts. $\left.\} \times \mathcal{F}_{p}^{\ell}\right)$ are mapped diffeomorphically onto the leaves of $\left(\mathrm{p}^{-1}(W),\left.\mathcal{F}^{\ell}\right|_{\mathrm{p}^{-1}(W)}\right)$.

The local closure of $\mathcal{F}^{\ell}$. Even though $\left(U_{p}, \mathcal{F}_{p}^{\ell}\right)$ is homogeneous for every $p \in B$, it might be the case that its leaves are not closed, which happens when the group $H_{p} \subseteq \mathrm{O}\left(U_{p}\right)$ defined in Proposition 2.10 is not closed. To obviate this problem we define a new foliation $\widehat{\mathcal{F}}^{\ell}$, called the local closure of $\mathcal{F}^{\ell}$, such that $\mathcal{F}^{\ell} \subset \widehat{\mathcal{F}}^{\ell} \subset \overline{\mathcal{F}^{\ell}}$ and whose restriction $\widehat{\mathcal{F}}_{p}^{\ell}$ to each p-fiber $U_{p}$ is homogeneous and closed.

Recall that $\mathcal{F}^{\ell}$ is defined by the orbits of the pseudogroup $\mathrm{D}$ of local diffeomorphisms, generated by the flows of linearized vector fields. For each $q \in U_{p}$, consider the closure $\bar{H}_{p}$ of $H_{p}$ in $\mathrm{O}\left(U_{p}\right)$, and define the $\widehat{\mathcal{F}}^{\ell}$-leaf $\widehat{L}_{q}$ through $q$ to be the D-orbit of $\bar{H}_{p} \cdot q$ :

$$
\widehat{L}_{q}=\left\{q^{\prime}=\Phi(h \cdot q) \mid \Phi \in \mathrm{D}, h \in \bar{H}_{p}\right\}
$$

Let $\sim$ denote the relation $q \sim q^{\prime}$ if and only if $q^{\prime}=\Phi(h \cdot q)$ for some $\Phi \in \mathrm{D}$ and $h \in \bar{H}_{p}$. In this way, the leaf of $\widehat{\mathcal{F}}^{\ell}$ through $q$ can be rewritten as $\left\{q^{\prime} \in U \mid q^{\prime} \sim q\right\}$. As for the other foliations, for every $p \in B$ we define $\left(U_{p}, \widehat{\mathcal{F}}_{p}^{\ell}\right)$ to be the partition of $U_{p}$ into the connected components, of the intersections of $U_{p}$ with the leaves in $\widehat{\mathcal{F}}^{\ell}$.

Proposition 6.2. The following hold:

(1) $\widehat{\mathcal{F}}^{\ell}$ is a well defined partition of $U$.

(2) For every $p \in B$ the leaves of $\widehat{\mathcal{F}}_{p}^{\ell}$ are the orbits of $\bar{H}_{p}$ on $U_{p}$.

Proof. 1. One must prove that the relation $\sim$ defined above is an equivalence relation. For this, notice that, since any $\Phi \in \mathrm{D}$ defines a foliated isometry between $\left(U_{p}, \mathcal{F}_{p}^{\ell}\right)$ and $\left(U_{\Phi(p)}, \mathcal{F}_{\Phi(p)}^{\ell}\right)$ for any $p \in B$, in particular it defines a foliated isometry between the respective closures $\left(U_{p}, \bar{H}_{p}\right)$ and $\left(U_{\Phi(p)}, \bar{H}_{\Phi(p)}\right)$. In particular, for any $h \in \bar{H}_{p}$ and $\Phi \in \mathrm{D}$, one has $h^{\prime}=\Phi \circ h \circ \Phi^{-1} \in \bar{H}_{\Phi(p)}$.

- Reflexivity of $\sim$ : if $q^{\prime} \sim q$ then $q^{\prime}=\Phi(h(q))$ for some $h \in \bar{H}_{p}$ and $\Phi \in \mathrm{D}$. Then $q^{\prime}=h^{\prime}(\Phi(q))$, where $h^{\prime}=\Phi \circ h \circ \Phi^{-1} \in \bar{H}_{\Phi(p)}$, and therefore $q=\Phi^{-1}\left(\left(h^{\prime}\right)^{-1} q^{\prime}\right)$, that means $q \sim q^{\prime}$.

- Transitivity of $\sim$ : if $q^{\prime} \sim q$ and $q^{\prime \prime} \sim q^{\prime}$ then $q^{\prime}=\Phi(h(q))$ and $q^{\prime \prime}=\Psi\left(g\left(q^{\prime}\right)\right)$ for some $h \in \bar{H}_{p}, g \in \bar{H}_{\Phi(p)}$, and $\Phi, \Psi \in \mathrm{D}$. Then $q^{\prime \prime}=(\Psi \circ \Phi)\left(\left(g^{\prime} \circ h\right)(q)\right)$, where $g^{\prime}=\Phi^{-1} \circ g \circ \Phi \in \bar{H}_{p}$, and therefore $q^{\prime \prime} \sim q$.

2. Let $L^{\prime}$ denote a leaf of $\widehat{\mathcal{F}}^{\ell}$. From (1), the intersection of $L^{\prime}$ with $U_{p}$ is a union of orbits of $\bar{H}_{p}$. On the other hand, we claim that the intersection $L^{\prime} \cap U_{p}$ consists of countably many orbits of $\bar{H}_{p}$, so that each connected component of such intersection must consists of a single $\bar{H}_{p}$-orbit. From the definition of $\widehat{\mathcal{F}}^{\ell}$, it is enough to prove that the subgroup $\mathrm{D}_{p} \subset \mathrm{D}$ of diffeomorphisms fixing $p$ moves every $\bar{H}_{p}$-orbit in $L^{\prime} \cap U_{p}$ to at most countably many orbits. For this, consider a piecewise smooth loop $\gamma:[0,1] \rightarrow L_{p}$ with $\gamma(0)=\gamma(1)=p$. Using linearized vector fields with $\gamma$ as integral curve, one can construct a continuous path $\Phi_{t}:[0,1] \rightarrow \mathrm{D}$ of diffeomorphisms such that $\Phi_{0}=i d_{U}$ and $\Phi_{t}(p)=\gamma(t)$, as described in [8, Cor. 7]. Fixing some $\bar{H}_{p}$-orbit $\mathcal{O}$ in $L^{\prime} \cap U_{p}$, its image $\Phi_{1}(\mathcal{O})$ is again some $\bar{H}_{p}$-orbit, which only depends on the class $[\gamma] \in \pi_{1}\left(L_{p}, p\right)$ and not on the actual path $\gamma$, nor on the 
specific choice of $\Phi_{t}$. This gives a map

$$
\partial: \pi_{1}\left(L_{p}, p\right) \rightarrow\left\{\bar{H}_{p^{-}} \text {orbits in } L^{\prime} \cap U_{p}\right\}
$$

This map admits a section, namely: for every orbit $\mathcal{O}^{\prime}$ in $L^{\prime} \cap U_{p}$, take a path $\gamma$ in $L^{\prime}$ from a point in a (fixed) orbit $\mathcal{O}$ to a point in $\mathcal{O}^{\prime}$. Under the projection $\mathrm{p}: U \rightarrow B$, the composition $\mathrm{p} \circ \gamma$ is a loop in $L_{p}$. The section of $\partial$ sends $\mathcal{O}^{\prime}$ to $[\mathrm{p} \circ \gamma] \in \pi_{1}\left(L_{p}, p\right)$. In particular, the map $\partial$ is surjective, and therefore the set of $\bar{H}_{p}$-orbits in $L^{\prime} \cap U_{p}$ has at most the cardinality of $\pi_{1}\left(L_{p}, p\right)$, which is at most countable since $L_{p}$ is a manifold.

As a corollary of Propositions 6.2 and 6.1, one gets the following:

Corollary 6.3. Let $(U, \mathcal{F})$ be a singular Riemannian foliation as in Section 4. let $\mathcal{F}^{\ell}$ be its linearized foliation and $\widehat{\mathcal{F}}^{\ell}$ the local closure. Then $\widehat{\mathcal{F}}^{\ell}$ is a singular foliation with complete leaves. Moreover, around each point $p \in B$ there is a neighbourhood $W$ of $p$ in $B$ such that $\left(\mathrm{p}^{-1}(W),\left.\widehat{\mathcal{F}}^{\ell}\right|_{\mathrm{p}^{-1}(W)}\right)$ is foliated diffeomorphic to a product

$$
\left(\mathbb{D}^{k} \times \mathbb{D}^{m-k} \times U_{p}, \mathbb{D}^{k} \times\{\text { pts. }\} \times\left\{\text { orbits of } \bar{H}_{p} \subseteq \mathrm{O}\left(U_{p}\right)\right\}\right),
$$

which can be given the structure of a singular Riemannian foliation.

Once it is shown that $\widehat{\mathcal{F}}^{\ell}$ is also a transnormal system with respect to some metric, then by the corollary above it is globally a singular Riemannian foliation.

\section{A NEW METRIC}

Let $\mathcal{T}, \mathcal{N}, \mathcal{K}$ be the distributions as in the previous section. Clearly, one has $T U=\mathcal{T} \oplus \mathcal{N} \oplus \mathcal{K}$.

Define now the new metric $\mathrm{g}^{\ell}$ on $U$, as the metric defined by the following properties:

- $\mathcal{T} \oplus \mathcal{N}$ and $\mathcal{K}$ are orthogonal with respect to $\mathrm{g}^{\ell}$.

- $\left.\mathrm{g}^{\ell}\right|_{\mathcal{T} \oplus \mathcal{N}}=\mathrm{p}^{*} \mathrm{~g}_{B}$, where $\mathrm{g}_{B}$ denotes the restriction of the original metric on $B$. In particular, $\mathcal{T}$ and $\mathcal{N}$ are also orthogonal to one another.

- For any $q \in U_{p}$, recall that $\mathcal{K}_{q}=T_{q} U_{p}$, and define $\left.\mathrm{g}^{\ell}\right|_{\mathcal{K}_{q}}=\mathrm{g}_{p}$ the flat metric on $U_{p}$ induced from $\exp _{p}: \nu_{p} B \rightarrow U_{p}$.

These conditions characterize the metric $\mathrm{g}^{\ell}$ uniquely. The most useful property of this metric is the following.

Proposition 7.1. The triples $\left(U, \mathrm{~g}^{\ell}, \mathcal{F}^{\ell}\right)$ and $\left(U, \mathrm{~g}^{\ell}, \widehat{\mathcal{F}}^{\ell}\right)$ are singular Riemannian foliations.

Proof. The arguments for $\mathcal{F}^{\ell}$ and $\widehat{\mathcal{F}}^{\ell}$ are ideantical, therefore we will only check the Proposition for $\left(U, \mathrm{~g}^{\ell}, \widehat{\mathcal{F}}^{\ell}\right.$ ) (which is the only case we need for the Main Theorem anyway). Moreover, the statement is local in nature, therefore it is enough to prove the statement on certain open sets covering the whole of $U$. For any point $p \in B$, let $W$ denote a neighbourhood of $p$ in $B$ and $\mathrm{p}^{-1}(W)$ a neighbourhood of $p$ in $U$. We need to check that $\left(\mathrm{p}^{-1}(W), \mathrm{g}^{\ell}, \widehat{\mathcal{F}}^{\ell}\right)$ is a singular Riemannian foliation. To prove this, we apply Proposition 2.14 of [2] which states that it is enough to check two conditions:

(1) $\left(\mathrm{p}^{-1}(W), g^{\prime}, \widehat{\mathcal{F}}^{\ell}\right)$ is a singular Riemannian foliation with respect to some Riemannian metric $g^{\prime}$. 
(2) For every stratum $\Sigma \subset \mathrm{p}^{-1}(W)$ (i.e. union of leaves of the same dimension), the restriction of $\widehat{\mathcal{F}}^{\ell}$ to $\Sigma$ is a (regular) Riemannian foliation.

The first condition is satisfied by Corollary 6.3. The second condition is equivalent to checking that, for every leaf $\widehat{L}_{q}$ of $\left.\widehat{\mathcal{F}}^{\ell}\right|_{\mathrm{p}^{-1}(W)}$ and every basic vector field $X$ along $\widehat{L}_{q}$ tangent to the stratum through $\widehat{L}_{q}$, the norm $\|X\|_{\mathrm{g} \ell}$ is constant along $\widehat{L}_{q}$.

By definition of the metric $g^{\ell}$, the space $\nu \widehat{L}_{q}$ is given by $\left.\mathcal{N}\right|_{\widehat{L}_{q}} \oplus\left(\nu \widehat{L}_{q} \cap \mathcal{K}\right)$. From Proposition 5.1, along $\widehat{L}_{q}$ the space $\mathcal{N}$ is spanned by linearized vector fields $X_{i}^{\ell}$, which are then $\mathrm{g}^{\ell}$-basic (i.e., foliated and $\mathrm{g}^{\ell}$-orthogonal to the leaves). In particular, any basic vector field $\bar{X}$ along $\widehat{L}_{q}$ splits as a sum $\bar{X}=\bar{X}_{1}+\bar{X}_{2}$, where $\bar{X}_{1}$ is tangent to $\mathcal{N}, \bar{X}_{2}$ is tangent to $\mathcal{N}^{\prime}:=\nu \widehat{L}_{q} \cap \mathcal{K}$, and $\mathrm{g}^{\ell}\left(\bar{X}_{1}, \bar{X}_{2}\right)=0$. Therefore, it is enough to check independently that for every basic vector field $\bar{X}$ along $\widehat{L}_{q}$, tangent to either $\mathcal{N}$ or $\mathcal{N}^{\prime}$, the norm of $\bar{X}$ is constant along $\widehat{L}_{q}$.

If $\bar{X}$ is tangent to $\mathcal{N}$, then by the construction in Proposition 5.1 it projects to some basic vector field $X$ along $\widehat{L}_{p} \subset B$. Since $\left(B, \mathrm{~g}_{B},\left.\mathcal{F}\right|_{B}\right)$ is a Riemannian foliation, the norm $\|X\|_{\mathrm{g}_{B}}$ is constant along $\widehat{L}_{p}$. By the construction of the metric $\mathrm{g}^{\ell}$, one has $\|\bar{X}\|_{\mathrm{g}^{\ell}}=\|X\|_{\mathrm{g}_{B}}$ and, therefore, the norm of $\bar{X}$ is constant along $\widehat{L}_{q}$.

If $\bar{X}$ is tangent to $\mathcal{N}^{\prime}$, then it is tangent to any fiber $U_{p^{\prime}}, p^{\prime} \in \widehat{L}_{p}$. The restriction $\left.\bar{X}\right|_{U_{p^{\prime}}}$ is a basic vector field of $\left(U_{p^{\prime}}, \widehat{\mathcal{F}}_{p^{\prime}}^{\ell}\right)$ along $\widehat{L}_{q} \cap U_{p^{\prime}}$, and therefore the norm $\left\|\left.\bar{X}\right|_{U_{p^{\prime}}}\right\|_{\mathrm{g}_{p^{\prime}}}$ is locally constant along $\widehat{L}_{q} \cap U_{p^{\prime}}$. By the construction of $\mathrm{g}^{\ell}$, it follows that $\left\|\left.\bar{X}\right|_{U_{p^{\prime}}}\right\|_{\mathrm{g} \ell}$ is also locally constant along each $\widehat{L}_{q} \cap U_{p^{\prime}}$. However, given two points $p^{\prime}, p^{\prime \prime} \in \widehat{L}_{p}$, and a vertical, foliated vector field $V^{\ell}$ whose flow $\Phi$ moves $p^{\prime}$ to $p^{\prime \prime}$, one also has that $\Phi$ moves $U_{p^{\prime}}$ isometrically to $U_{p^{\prime \prime}}$, and $\left.\bar{X}\right|_{U_{p^{\prime}}}$ to $\left.\bar{X}\right|_{U_{p^{\prime \prime}}}$. In particular, $\left\|\left.\bar{X}\right|_{U_{p^{\prime}}}\right\|_{\mathrm{g}^{\ell}}=\left\|\left.\bar{X}\right|_{U_{p^{\prime}}}\right\|_{\mathrm{g}_{p^{\prime}}}$ does not really depend on the point $p^{\prime} \in \widehat{L}_{p}$, and it is actually constant along the whole leaf $\widehat{L}_{q}$.

With this in place, one can finally prove the Main Theorem:

Proof of the Main Theorem. Let $U$ be an $\epsilon$-tubular neighbourhood around the closure $\bar{L}$ of a leaf $L \in \mathcal{F}$. Letting $B=\bar{L}$, we are under the assumptions of Section 4. In particular, it is possible to define the linearized foliation $\mathcal{F}^{\ell}$ on $U$, its local closure $\widehat{\mathcal{F}}^{\ell}$, and the metric $\mathrm{g}^{\ell}$ as in Proposition 7.1. It is clear by construction that $\left.\widehat{\mathcal{F}}^{\ell}\right|_{L}=\left.\mathcal{F}\right|_{L}$ and that the closure of $\widehat{\mathcal{F}}^{\ell}$ is contained in the closure of $\mathcal{F}$. Moreover, by Corollary 6.3 the foliation $\left(U, \mathrm{~g}^{\ell}, \widehat{\mathcal{F}}^{\ell}\right)$ is, locally around each point, foliated diffeomorphic to the orbit like foliation $\left(\mathbb{D}^{k} \times \mathbb{D}^{m-k} \times U_{p}, \mathbb{D}^{k} \times\{\right.$ pts. $\left.\} \times \widehat{\mathcal{F}}_{p}^{\ell}\right)$. By Proposition 2.9. the foliation $\left(U, \mathrm{~g}^{\ell}, \widehat{\mathcal{F}}^{\ell}\right)$ is orbit like as well, and this concludes the proof.

\section{REFERENCES}

1. M. M. Alexandrino, Proofs of conjectures about singular Riemannian foliations, Geom. Dedicata 119 (2006) no. 1, 219-234.

2. M. M. Alexandrino Desingularization of singular Riemannian foliation, Geom. Dedicata 149 (2010) 397-416.

3. M. M. Alexandrino and A. Lytchak, On smoothness of isometries between orbit spaces, Riemannian geometry and applications-Proceedings RIGA (2011), 17-28, Ed. Univ. Bucureşti. 
4. M. M. Alexandrino and M. Radeschi, Smoothness of isometric flows on orbit spaces and applications to the theory of foliations, Transf. Groups, DOI: 10.1007/s00031-016-9386-5 (2016), $1-26$.

5. J. Bolton, Transnormal systems, Q. J. of Math. 24, no.1 (1973) 385-395.

6. J. Cheeger and D. Gromoll, On the Structure of Complete Manifolds of Nonnegative Curvature, Ann. of Math. 96 (1972), no. 3, 413-443.

7. A. Lytchak and M. Radeschi, Algebraic nature of singular Riemannian foliations in spheres, to appear in J. Reine Ang. Math., DOI: https://doi.org/10.1515/crelle-2016-0010.

8. R. Mendes and M. Radeschi Smooth basic functions, preprint: arXiv:1511.06174 [math.DG].

9. P. Molino, Riemannian foliations, Progress in Mathematics 73, Birkhäuser, Boston (1988).

10. P. Molino, Orbit-like foliations, Proceedings of Geometric study of Foliations, held in Tokyo, November 1993, ed. T. Mizutani et al. World Scientific, Singapore (1994), 97-119.

11. S. Kobayashi and K. Nomizu, Foundations of Differential geometry, Vol. I, Interscience tracts in pure and applied mathematics, Interscience Editor, New York (1963).

12. M. Radeschi, Clifford algebras and new singular Riemannian foliations in spheres, Geom. Funct. Anal 24 (2014) 5, 1660-1682.

13. H. J. Sussmann, Orbits of families of vector fields and integrability of distributions. Trans. of the Am. Math. Soc., 180 (1973), 171-188.

14. D. Toeben, Parallel focal structure and singular Riemannian foliations, Trans. Am. Math. Soc. 358, 1677-1704 (2006).

15. B. Wilking, A Duality Theorem for Riemannian Foliations in Nonnegative Sectional Curvature, Geom. Funct. Anal. 17 (2007), no. 4, 1297-1320.

Marcos M. Alexandrino

Universidade de São Paulo, Instituto de Matemática e Estatística, Rua do Matẽo 1010,05508 090 SÃo PaUlo, Brazil

E-mail address: marcosmalex@yahoo.de, malex@ime.usp.br

MARCO RADESCHI

Mathematisches Institut, WWU Münster, Einsteinstr. 62, MÜnster, Germany.

E-mail address: mrade_02@uni-muenster.de 